\title{
Adaptive Resource Allocation in Wireless Relay Networks
}

\author{
Tobias Renk \\ Institut für Nachrichtentechnik \\ Universität Karlsruhe (TH) \\ Germany \\ Email: renk@int.uni-karlsruhe.de
}

\author{
Dimitar Iankov \\ Institut für Nachrichtentechnik \\ Universität Karlsruhe (TH) \\ Germany \\ Email: iankov@int.uni-karlsruhe.de
}

\author{
Friedrich K. Jondral \\ Institut für Nachrichtentechnik \\ Universität Karlsruhe (TH) \\ Germany \\ Email: fj@int.uni-karlsruhe.de
}

\begin{abstract}
We consider a wireless relay network in a Rayleigh fading scenario where transmission power as well as transmission time per user are optimized. The criterion of optimization is the capacity. We apply an optimization algorithm based on Brent's method. Hence, we employ parabolic interpolation for finding the optimum whenever possible in order to achieve a faster convergence. If parabolic interpolation is not suitable, we use golden section search which is a robust root-finding method. Optimization takes place in two steps. First, we optimize power allocation and consider time as parameter. Second, time is optimized. We demonstrate that, depending on the cooperation strategy, remarkable capacity gains over direct transmission can be achieved by multi-routing. However, for a high overall transmission power, direct transmission outperforms multi-hopping. Generally, capacity gains increase with decreasing overall system power, which demonstrates that relaying is beneficial for low overall transmission powers.
\end{abstract}

\section{INTRODUCTION}

Recently an enormous growth in the interest of relay networking can be noticed. The basic aim of relaying is to achieve spatial diversity in order to combat signal fluctuations at the receiver. For that purpose, several mobile users pool their resources in order to create a virtual antenna array. Suitable cooperation strategies lead to great improvements with respect to maximal data rate, error probability, transmission range, and outage probability [1].

The classical concept of the relay channel was introduced by van der Meulen in 1968 [2]. A rigorous informationtheoretic analysis has been exposed by Cover and El Gamal in 1979 [3]. It was not until 2003 when the interest in cooperation began to rise again [4], [5]. The main reason for this might be limitations in hardware and chip design which made cooperation impossible. Since then, cooperation in wireless networks has become one of the most important parts in research. Major reason for this is that the conservative way to transmit data is not sufficient anymore for the needs of future multi-media applications due to an increasing number of users and the existence of more resource demanding services. These demands require higher data rates compared to single-hop transmission with one antenna at each node. An alternative solution to this problem is the use of multiple-input multiple-output (MIMO) systems [6], [7]. However, due to size, cost, and/or hardware limitations, mobile nodes can only be equipped with a determined number of antennas.
Hence, we use a network scenario in this paper where each node is equipped with one antenna and optimize resource allocation of power and time. Hasna and Alouini also considered power allocation for relayed transmission in [8] where outage probability has been used as optimization criterion. In [9] power and time have been optimized together for an opportunistic protocol and a long-term power constraint. We present an optimization algorithm that is suitable for practical implementation. We will see that our results match perfectly with those derived by Gündüz and Erkip in [9].

The paper is organized as follows. Section II describes the network and the path-loss model. In Section III optimization of resource allocation is dealt with. We first describe the optimization problem and then introduce an optimization algorithm based on Brent's method [10], [11]. Section IV is concerned with the simulations and results are presented. Finally, Section V concludes the paper.

\section{NETWORK MODEL}

We consider the network depicted in Fig. 1. It consists of one source $S$, one relay $R$, and one destination $D$. The channel gains $h_{i}, i \in\{\mathrm{sd}, \mathrm{sr}, \mathrm{rd}\}$, are subject to a Rayleigh fading profile with variances $\sigma_{i}^{2}$. Hence, the probability density function of $\left|h_{i}\right|$ is given by

$$
f_{\left|h_{i}\right|}\left(\left|h_{i}\right|\right)=\frac{2\left|h_{i}\right|}{\sigma_{i}^{2}} \exp \left(-\frac{\left|h_{i}\right|}{\sigma_{i}^{2}}\right),
$$

where $\sigma_{i}^{2}=\mathbb{E}\left(\left|h_{i}\right|^{2}\right)$. Thus, $\left|h_{i}\right|^{2}$ follow an exponential distribution with mean value $\sigma_{i}^{2}$ :

$$
f_{\left|h_{i}\right|^{2}}\left(\left|h_{i}\right|^{2}\right)=\frac{1}{\sigma_{i}^{2}} \exp \left(-\frac{\left|h_{i}\right|^{2}}{\sigma_{i}^{2}}\right)
$$

We use a common path-loss model, where the channel variances are inverse proportional to the distance between two nodes. We can write $\sigma_{i}^{2} \propto d_{i}^{-\alpha}$, where $d_{i}$ is the distance between two users and $\alpha$ denotes the path-loss exponent. For cellular mobile networks, $\alpha$ takes values between 3 and 5 . The distance $S-D$ is normalized to 1 . We furthermore assume the relay to be placed on a straight line between $S$ and D. Hence, $d_{\mathrm{sr}}=1-d_{\mathrm{rd}}$ (see Fig. 2). We have

$$
\sigma_{\mathrm{sd}}^{2}=1, \quad \sigma_{\mathrm{sr}}^{2}=\frac{1}{d_{\mathrm{sr}}^{\alpha}}, \quad \sigma_{\mathrm{rd}}^{2}=\frac{1}{\left(1-d_{\mathrm{sr}}\right)^{\alpha}} .
$$




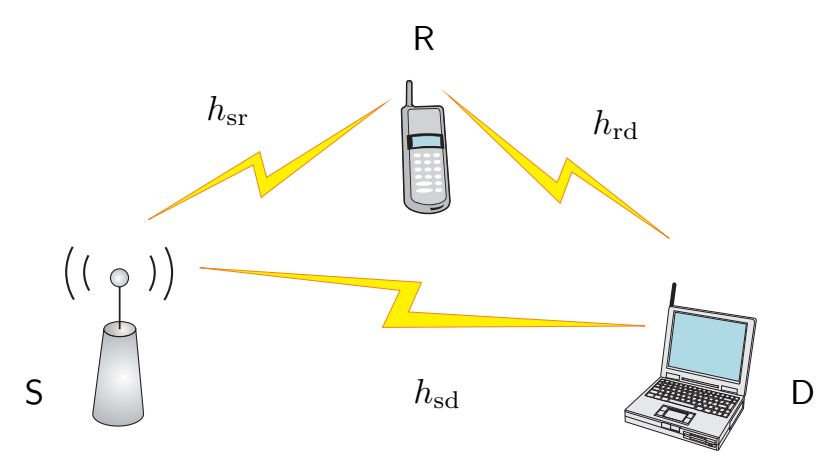

Fig. 1. Network consisting of source $S$, relay R, and destination D. Channel gains are represented by $h_{\mathrm{sd}}, h_{\mathrm{sr}}$, and $h_{\mathrm{rd}}$.

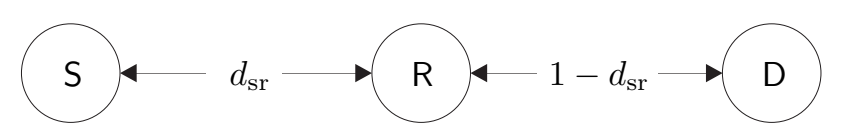

Fig. 2. One-dimensional network geometry. The distance between S and D is normalized to $d_{\mathrm{sd}}=1$. Furthermore, $d_{\mathrm{sr}}=1-d_{\mathrm{rd}}$.

On each channel, additive white Gaussian noise is added. Noise realizations are considered to be independent and follow $\mathcal{C N}(0,1)$. A network realization is described by the triple $\left(\left|h_{\mathrm{sd}}\right|^{2},\left|h_{\mathrm{sr}}\right|^{2},\left|h_{\mathrm{rd}}\right|^{2}\right)$. We assume full channel state information at the receivers and partial channel state information at the transmitters. The relay operates in a half-duplex mode so that it cannot receive and transmit simultaneously. Decodeand-forward is used at the relay, which means that the relay decodes its receive signal and encodes it again before retransmission. An allocation strategy $(\mathbf{P}, \mathbf{t})$ is described by the power allocation vector $\mathbf{P}=\left[P_{\mathrm{S}}, P_{\mathrm{r}}\right]^{T}$ and the time allocation vector $\mathbf{t}=[t, 1-t]^{T}$, where $P_{\mathrm{s}}$ denotes the source power, $P_{\mathrm{r}}$ denotes the relay power, and $t$ is the time fraction used for source transmission (i.e., $1-t$ is used for relaying).

\section{Resource Allocation}

In this section we describe an algorithm where power and time allocation are optimized to achieve the maximal capacity depending on a power constraint and channel gains. We first describe the optimization problem and then present an optimization algorithm based on Brent's method.

\section{A. Optimization Problem}

The overall transmission power $P_{\text {tot }}$ is constrained like follows:

$$
P_{\text {tot }} \triangleq t P_{\mathrm{s}}+(1-t) P_{\mathrm{r}}
$$

Here, $t$ describes the time ratio that is reserved for source transmission, $P_{\mathrm{s}}$ is the source power, and $P_{\mathrm{r}}$ is the relay power. The overall aim is to derive an optimal resource allocation so that the capacity is maximized:

$$
\begin{gathered}
C^{*}=\max _{t} \max _{P_{\mathrm{s}}}\left\{C\left(P_{\text {tot }}, \mathbf{h}, t\right): t P_{\mathrm{s}}+(1-t) P_{\mathrm{r}}=P_{\text {tot }}\right\} \\
\text { subject to }=\left\{\begin{array}{l}
P_{\mathrm{s}} \in\left[0, P_{\text {tot }} / t\right] \\
t \in(0,1]
\end{array}\right.
\end{gathered}
$$

where $\mathbf{h}$ represents the triple $\left(\left|h_{\mathrm{sd}}\right|^{2},\left|h_{\mathrm{sr}}\right|^{2},\left|h_{\mathrm{rd}}\right|^{2}\right)$. The optimization algorithm consists of two subsequent steps. First power allocation is optimized, where overall transmission power $P_{\text {tot }}$ and time $t$ are considered as parameters. In the second step time allocation is optimized and the pair $\left(P^{*}, t^{*}\right)$ which maximizes capacity is determined. Here, we have an optimization on the closed interval $t \in(0,1)$. The function values are provided by the power allocation in the first step.

\section{B. Optimization Algorithm}

For both optimization steps, i.e., optimization of power and time allocation, we apply an algorithm based on Brent's method [10], [11].

1) Description: Brent's method is a root-finding algorithm in numerical analysis that does not require any derivations of functions. We use a similar algorithm by combining golden section search and parabolic interpolation. The advantage of this combination is that we have a robust optimization algorithm due to the reliability of the golden section search and a faster convergence (compared to the golden section search alone) due to the use of parabolic interpoaltion whenever possible. There are several requirements for the usage of such an algorithm:

- The function that is optimized has to be continuous with respect to the optimization variable.

- Optimization can only be done with respect to one variable.

- The function has to be unimodular in order to be able to find the extreme value. If there are more than one extreme values, then only one extreme value will be found. However, this has not to be the global optimum.

We will later see that these requirements are met when we maximize capacity and thus the applied optimization algorithm provides reliable results.

2) Golden Section Search: Consider a unimodal function on the closed (bracketing) interval $(a, b)$. Hence, there exists exactly one $p \in(a, b)$ so that the function is decreasing in $(a, p]$ and increasing in $[p, b)$ (or vice versa). We next choose an initial value of $v=a+c(b-a)$ with

$$
c=\frac{1}{\phi^{2}}=\frac{3-\sqrt{5}}{2} \approx 0.382,
$$

where $\phi$ is the golden ratio

$$
\phi=\frac{1+\sqrt{5}}{2} .
$$

In the next iteration, the bracketing interval is $(v, b)$ and this interval is now divided with respect to the golden ratio again. Say we get the point $u$. Now, if $f(u)<f(v)$, the new bracketing interval will be $(u, b)$. Otherwise, for $f(u)>f(v)$, the new bracketing interval is $(a, u)$. Golden section search reliably finds the optimum of a function, even if this function behaves "uncooperatively" (which means that it progresses unsteadily). 
TABLE I

FIVE ITERATIONS OF THE OPTIMIZATION ALGORITHM (CF. FIG. 3).

$\begin{array}{cccl}\# & x & f(x) & \text { Procedure } \\ 1 & 0.76 & -1.81 & \text { initial } \\ 2 & 1.23 & -2.13 & \text { golden } \\ 3 & 1.52 & -2.27 & \text { golden } \\ 4 & 1.71 & -2.02 & \text { golden } \\ 5 & 1.44 & -2.24 & \text { parabolic }\end{array}$

3) Parabolic Interpolation: Parabolic interpolation converges much faster than golden section search. With parabolic interpolation the optimum of a smooth function can be found by approximating the function by a parabola. We consider three points $(u, f(u)),(v, f(v))$, and $(w, f(w))$ of the function $f$. These three points clearly define a parabola. The minimum $x^{*}$ of this parabola is given by

$x^{*}=v-\frac{1}{2} \frac{(v-u)^{2}(f(v)-f(w))-(v-w)^{2}(f(v)-f(u))}{(v-u)(f(v)-f(w))-(v-w)(f(v)-f(u))}$.

We use parabolic interpolation whenever possible. If an approximation of the function $f$ by a parabola is not acceptable, we apply the golden section search. Therefore, we exploit two advantages. first, the golden section search ensures that we find an optimum. Second, the use of parabolic interpolation leads to a faster convergence.

We now give a simple example that shows the operating mode of the optimization algorithm. The function

$$
\begin{aligned}
-f(x)= & -\frac{1}{2} \min \left\{\log _{2}(1+b x),\right. \\
& \left.\log _{2}(1+a x)+\log _{2}(1+c z)\right\}
\end{aligned}
$$

is minimized with respect to the variable $x$. We choose $a=1$, $b=14.78, c=17.35$, and $z=2(1-x)$. We will later see that $f(x)$ indeed shows similarities to the achievable rate of a multi-route relay network with decode-and-forward at the relay. Fig. 3 shows the function and indicates the first 5 iterations. We can see that $f(x)$ is unimodal and that there exists exactly one extreme value. The point 1 is the initial point that has been chosen with respect to the golden ratio. The points 2,3 , and 4 have been found by the golden section search. For point 5 parabolic interpolation has been used. Values for the first five iterations are given in Tab. I. Optimization surely doe not stop here, but continues until a certain termination criterion is met, e.g., the change of the functional value $f(x)$ is lower than $10^{-4}$ for subsequent iterations.

\section{Capacity}

In this section we give equations on the capacities of two cooperation strategies. The basic transmission scheme is illustrated in Fig. 4. The first phase is reserved for source transmission and the second phase for relay transmission.

First, consider multi-routing. Here, the source sends information in the first transmission phase to the relay and the destination. In the second phase the relay transmits a newly encoded version of the source signal to the destination, thus,

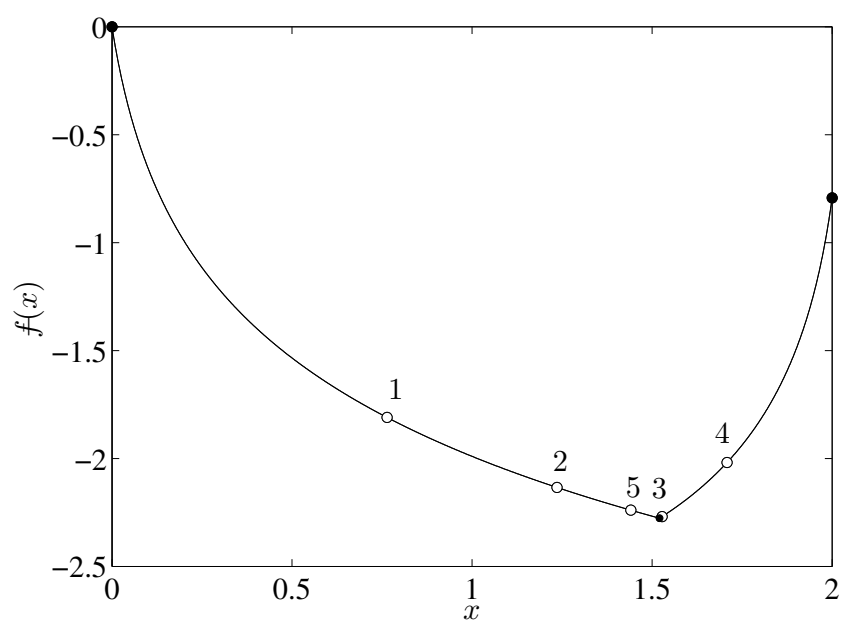

Fig. 3. Operating mode of the optimization algorithm. Point 1 is the initial value. Points 2,3 , and 4 have been found by golden section search, whereas point 5 has been found by parabolic interpolation.

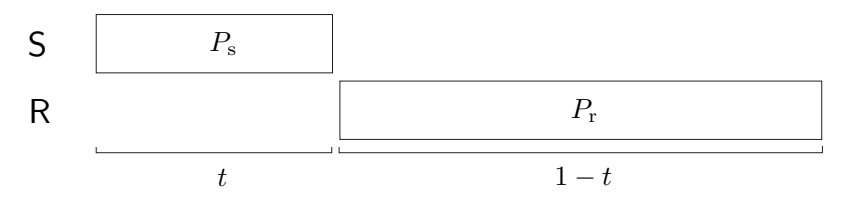

Fig. 4. Basic transmission scheme. The first phase of duration $t$ is reserved for source transmission and the second phase for relay transmission.

creating diversity gains. Source and relay use independently created Gaussian codebooks. Consequently, the destination must know both codebooks. The achievable rate for this transmission scheme becomes

$$
\begin{aligned}
& C_{\mathrm{MR}}=\min \left\{t \log _{2}\left(1+\left|h_{\mathrm{sr}}\right|^{2} P_{\mathrm{s}}\right)\right. \\
& \left.\quad t \log _{2}\left(1+\left|h_{\mathrm{sd}}\right|^{2} P_{\mathrm{s}}\right)+(1-t) \log _{2}\left(1+\left|h_{\mathrm{rd}}\right|^{2} P_{\mathrm{r}}\right)\right\}
\end{aligned}
$$

where the first expression in the min-function describes the maximal rate at which the relay can decode the source signal and the second expression is the maximal rate at which the destination can decode the source and the relay signal. We see that the use of independent codebooks leads to an accumulation of mutual information at the destination.

Second, consider multi-hopping. The difference between multi-hopping and multi-routing is that for multi-hopping there is no direct path from source to destination. In the first phase the source transmits only to the relay and in the second phase the relay transmits to the destination. In that case the destination only has to know the codebook from the relay. Accordingly, we get an achievable rate of

$C_{\mathrm{MH}}=\min \left\{t \log _{2}\left(1+\left|h_{\mathrm{sr}}\right|^{2} P_{\mathrm{s}}\right),(1-t) \log _{2}\left(1+\left|h_{\mathrm{rd}}\right|^{2} P_{\mathrm{r}}\right)\right\}$,

whose maximum for $t=1 / 2$ is clearly limited by the weakest channel in the network. (For a proof of this see [12], Ch. 2.3.) Our aim is to find an optimal allocation of power and time 
so that capacity is maximized (cf. (4)):

$$
C_{\{\mathrm{MH}, \mathrm{MR}\}}^{*}=\max _{t} \max _{P_{\mathrm{s}}} C_{\{\mathrm{MH}, \mathrm{MR}\}}
$$

The parameters are the relay location $d_{\mathrm{sr}} \in(0,1)$ and the pathloss exponent $\alpha$. As explained previously, we first optimize power allocation and consider $t$ as parameter. In the next step, $t$ is optimized. For both optimization tasks we apply the presented algorithm based on Brent's method.

\section{Simulations}

In this section we present the simulation results. Fig. 5 illustrates the optimal power allocation over the overall transmission power

$$
P^{*}=\frac{t^{*} P_{\mathrm{s}}}{P_{\mathrm{tot}}}
$$

for the source when the relay is located at $d_{\mathrm{sr}}=0.3$ (this already includes optimal time allocation as well). The power allocated to the source for the multi-route network is always higher than that for the multi-hop scenario. This is due to the fact that for multi-hopping there is no direct connection from $\mathrm{S}$ to D. Hence, it suffices to allocate less power to the source in order to achieve a non-outage transmission to the relay. For the relay power the situation is vice versa. Since there is no signal from the source to the destination for the case of multihopping, the relay transmission has to be much more reliable compared to multi-routing. Therefore, more power is allocated to the relay.

In Fig. 6 the optimal time allocation $t^{*}$ over the overall transmission power is depicted. We see that for small values of $P_{\text {tot }}$ there is no significant difference between multi-routing and multi-hopping with $t^{*}$ being slightly bigger for multihopping. For $P_{\text {tot }} \approx 5 \mathrm{~dB}$ both curves intersect. From that point on the difference between $t^{*}$ of multi-routing and multihopping increases for increasing values of $P_{\text {tot }}$.

In order to see the gains that we achieve over direct transmission in terms of capacity, we define the capacity gain as

$$
G\left(P_{\mathrm{tot}}, d_{\mathrm{sr}}\right) \triangleq 10 \log _{10}\left(\frac{C_{\{\mathrm{MH}, \mathrm{MR}\}}^{*}\left(P_{\mathrm{tot}}, d_{\mathrm{sr}}\right)}{C_{\mathrm{DT}}\left(P_{\mathrm{tot}}\right)}\right)
$$

In (12) $C_{\mathrm{DT}}\left(P_{\text {tot }}\right)$ is given by

$$
C_{\text {DT }}\left(P_{\text {tot }}\right)=\log _{2}\left(1+\left|h_{\text {sd }}\right|^{2} P_{\text {tot }}\right) \text {. }
$$

The results are shown in Fig. 7. For instance, consider a multi-route scenario with $P_{\text {tot }}=1 \mathrm{~dB}$ and a relay location of $d_{\mathrm{sr}}=0.3$. Then for $t^{*}=0.31$ and $P^{*}=0.29$ a gain of approximately $2.7 \mathrm{~dB}$ can be achieved in capacity. We furthermore see three interesting facts. First, multi-routing always performs better than multi-hopping. This is due to the fact that multi-hopping does not create any kind of spatial diversity in contrast to multi-routing. Second, multi-routing achieves always a gain over direct transmission. Third, this is, however, not always the case for multi-hopping. It can be seen that for $P_{\mathrm{tot}}=10 \mathrm{~dB}$ and $d_{\mathrm{sr}} \in(0,0.2)$ and $d_{\mathrm{sr}} \in(0.8,1)$, respectively, direct transmission outperforms multi-hopping.

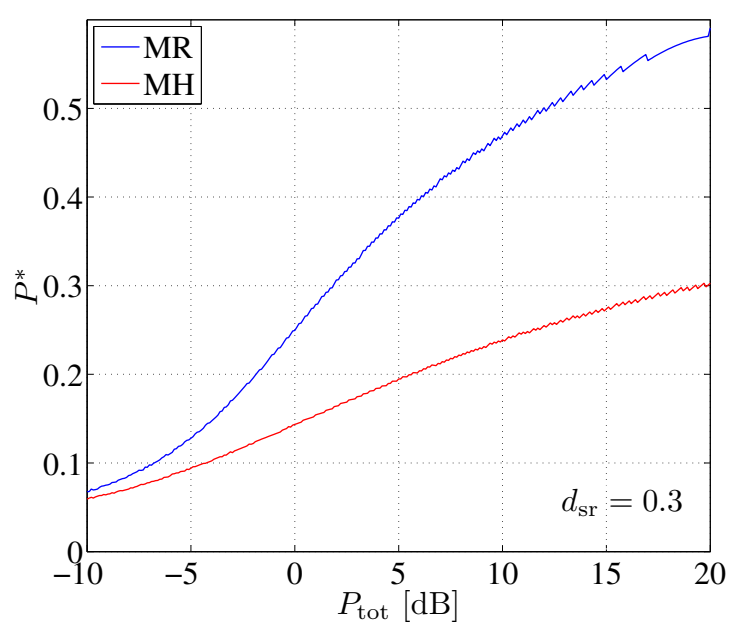

Fig. 5. Optimal power allocation $P^{*}=t^{*} P_{\mathrm{S}} / P_{\text {tot }}$ for the source. Distance $\mathrm{S}-\mathrm{R}$ is $d_{\mathrm{sr}}=0.3$.

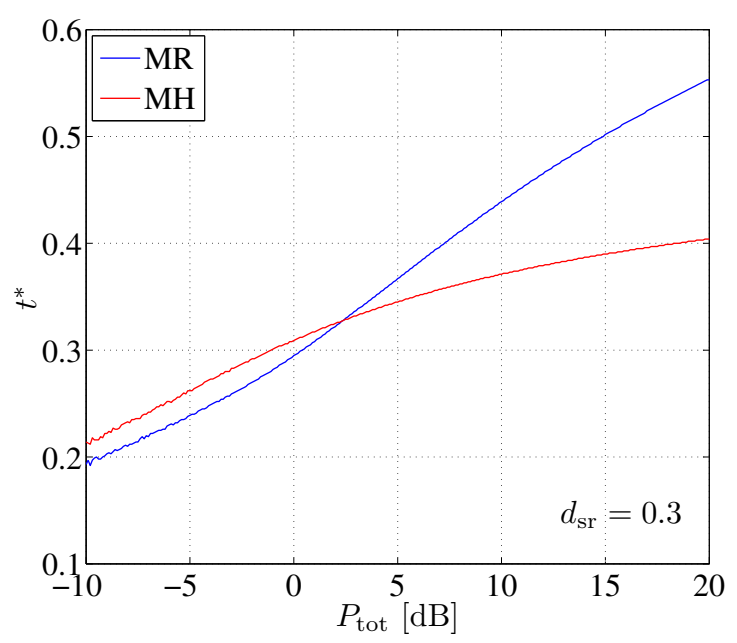

Fig. 6. Optimal time allocation $t^{*}$ for the source. Distance $\mathrm{S}-\mathrm{R}$ is $d_{\mathrm{sr}}=0.3$.

The reason for this is quite obvious. When the relay is closely located either to the source or the destination, one transmission distance for multi-hopping is in the range of the source-todestination distance and separation of power does not really make sense.

\section{CONCLUSIONS}

We proposed a combined optimization strategy for adaptive resource allocation in a wireless relay network. Allocation of transmission power and time per mobile user have been optimized in order to achieve a maximal overall capacity. We presented a two-step optimization algorithm, where we first optimize power and in a subsequent step optimize time. The algorithm is based on Brent's method, a well-known rootfinding algorithm in numerical analysis without the need of derivations. We have shown that dependent on the relay location and the overall system power $P_{\text {tot }}$ remarkable gains can be 


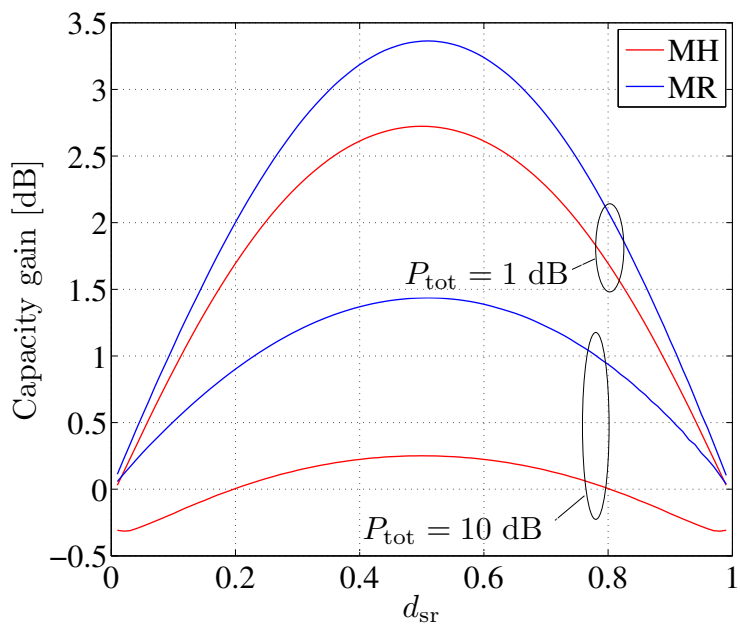

Fig. 7. Capacity gain of multi-route and multi-hop over direct transmission when optimized power allocation $P^{*}$ and optimized time allocation $t^{*}$ are used.

achieved by multi-routing and multi-hopping in comparison to direct transmission. However, for a higher overall transmission power there are relay locations where direct transmission outperforms multi-hopping. The reason for this is that multihopping, in contrast to multi-routing, does not create spatial diversity in the wireless network. Generally, we can state that capacity gains increase with decreasing overall system power. This clearly shows that relaying is beneficial for low overall transmission powers.

\section{REFERENCES}

[1] T. Renk, H. Jäkel, C. Klöck, and F. K. Jondral, "General expression of outage probability in cooperative networks," European Transactions on Telecommunications, accepted for publication.

[2] E. van der Meulen, "Transmission of information in a t-terminal discrete memoryless channel," Department of Statistics, University of California, Berkeley, CA, Technical Report, 1968.

[3] T. Cover and A. El Gamal, "Capacity theorems for the relay channel," IEEE Transactions on Information Theory, vol. 25, no. 5, pp. 572-584, September 1979.

[4] A. Sendonaris, E. Erkip, and B. Aazhang, "User cooperation diversity - part I: System description," IEEE Transactions on Communications, vol. 51, pp. 1927-1938, 2003.

[5] — - "User cooperation diversity - part II: Implementation aspects and performance analysis," IEEE Transactions on Communications, vol. 51, pp. 1939-1948, 2003.

[6] İ. E. Telatar, "Capacity of multi-antenna gaussian channels," Bell Labs Journal, vol. 10, no. 6, November 1999

[7] G. Foschini and M. J. Gans, "On limits of wireless communications in a fading environment when using multiple antennas," Wireless Personal Communications, Kluwer Academic Publishers, vol. 6, pp. 311-335, 1998.

[8] M. O. Hasna and M.-S. Alouini, "Optimal power allocation for relayed transmissions over rayleigh-fading channels," IEEE Transactions on Wireless Communications, vol. 3, no. 6, pp. 1999-2004, November 2004.

[9] D. Gündüz and E. Erkip, "Opportunistic cooperation by dynamic resource allocation," IEEE Transactions on Wireless Communications, vol. 6, no. 4, pp. 1446-1454, April 2004.

[10] R. P. Brent, Algorithms for Minimization Without Derivatives. Englewood Cliffs, New Jersey: Prentice-Hall, 1973.

[11] D. Anderson, "Algorithms for minimization without derivatives," IEEE Transactions on Automatic Control, vol. 19, pp. 632-633, October 1974.

[12] R. Gallager, Information Theory and Reliable Communication. John Wiley \& Sons, Inc., New York London Sydney Toronto, 1968. 